\title{
SEGURANÇA OU INSEGURANÇA DA SAÚDE MUNDIAL NA ÁFRICA? MAIS SAÚDE PARCIAL DO QUE SAÚDE GLOBAL*
}

\author{
Dominique Kerouedan \\ Professora da Paris School of International Affairs (PSIA - Sciences Po Paris), Cátedra \\ Geopolítica da Saúde Mundial do Collège de France (2012-2013). Paris, França. \\ Email<d.kerouedan@skynet.be>
}

http://dx.doi.org/10.1590/ 0102-6445047-076/98

A situação sanitária dos países da África continua dominada por doenças infecciosas e tropicais (ONU, 2012), pela penúria de profissionais da área e por parcos financiamentos. É confrontada ainda à crescente onda de doenças crônicas (câncer, diabetes, doenças cardiovasculares, respiratórias e mentais, além de deficiências), cujas consequências sanitárias não foram mensuradas no contexto atual de crescimento demográfico sem precedentes, com implicações diretas sobre o desenvolvimento desses países. A região francófona da África, por exemplo, atinge a mais alta taxa de fecundidade do mundo. O continente africano conta com mais da metade dos óbitos mundiais de mulheres grávidas e de mortalidade por aborto. As taxas de mortalidade infantil continuam elevadas, embora tenham decrescido (Balique, 2011).

Nos últimos trinta anos, três quartos das novas infecções por HIV notificadas no mundo ocorreram na África, como atestam os relatórios anuais do Programa Conjunto

\footnotetext{
* Tradução de Deisy de Freitas Lima Ventura, professora do Instituto de Relações Internacionais e da Faculdade de Saúde Pública da Universidade de São Paulo.
} 
das Nações Unidas sobre HIV/Aids (Unaids, 2013). Ainda longe de ter vencido a pandemia mais mortífera do século XX, o continente entra em uma nova fase de transição demográfica e epidemiológica. A urbanização galopante se faz acompanhar da transformação do modo de vida dos habitantes, expostos a novos riscos como sedentarismo, obesidade, uso nocivo do tabaco, do álcool e outras drogas. Pouco se leva em conta os efeitos da instabilidade política e dos conflitos armados sobre a saúde mental da população, em particular das crianças, assim como seus efeitos sobre a propagação da aids e sua feminização, com as agressões sexuais de meninas e mulheres que as expõe à infecção por HIV (ACSI, 2010).

Em resposta à evolução da situação sanitária e social dos africanos, buscando enfrentar as disfunções que acometem os seus sistemas de saúde, as respostas da cooperação sanitária internacional diversificaram-se ao longo das duas 48 últimas décadas. Ao lado da Organização Mundial da Saúde (OMS), da ajuda estatal bilateral clássica e de algumas organizações não governamentais (ONGs), a paisagem institucional da ajuda ao desenvolvimento diversificou-se na década de 1990 com a entrada em cena do Banco Mundial e da Comissão Europeia. Privilegiando até então relações econômicas e comerciais com os Estados da África, do Caribe e do Pacífico (ACP), o Fundo Europeu de Desenvolvimento passou a financiar programas de desenvolvimento humano. Três eventos centrais encontram-se na origem do envolvimento dessas duas figuras do desenvolvimento com a saúde: (i) a propagação da aids e suas repercussões sobre as economias africanas; (ii) os efeitos das políticas de ajustamento estrutural dos anos 1980 sobre os setores sociais e o acesso à saúde, quando as instituições de Bretton Woods foram severamente criticadas, a ponto de o Banco Mundial publicar, em 1993, um relatório convidando a "investir na saúde" (Banco Mundial, 1993); (iii) a desvalorização 
do franco das ex-colônias francesas da África (CFA), em janeiro de 1994, que obrigou a Comissão Europeia a compensar a fragilização da capacidade desses países africanos de importar medicamentos produzidos no hemisfério Norte, especialmente pela indústria europeia. A reforma dos sistemas de saúde exigida pelos governos, na realidade pelas instituições internacionais, foi financiada pelo Banco Mundial e pela Comissão Europeia, respectivamente por empréstimos e por doações ao longo dos anos na década de 1990, e também por meio da iniciativa de redução da dívida intitulada Países Pobres Muito Endividados (PPME), cujos financiamentos apoiam a realização de planos estratégicos nacionais de luta contra a pobreza, à época, paradigmas do desenvolvimento.

Desde meados dos anos 1990, a epidemia de aids explodiu especialmente na África Austral, mas não apenas lá. A pandemia escapou às estratégias nacionais e às instituições internacionais dela encarregadas. A liderança da OMS, autoridade sanitária mundial que havia instituído em 1987 o Programa Mundial de Aids (Global Aids Programe), encontrava-se fragilizada, tanto internamente em relação ao sistema das Nações Unidas como em escala internacional. Seguiu-se a criação, em 1996, do Programa Conjunto das Nações Unidas para Luta contra a Aids (Unaids), associando uma dezena de agências das Nações Unidas com o Banco Mundial. Mas a propagação rápida da pandemia continua desafiando os Estados e as instituições internacionais. As taxas de prevalência de infecção por HIV observadas entre os militares de diversos países da África Subsahariana, constantemente superiores aos da população em geral, chamaram a atenção das autoridades sul-africanas e norte-americanas que aí perceberam uma ameaça para a segurança dos Estados Unidos - num contexto em que Bill Clinton havia evocado, desde 1996, uma estratégia de segurança nacional mais centrada nos riscos infecciosos. O diretor da 
Unaids à época, Peter Piot (2011), explicou a quem quisesse escutar: "falta apenas o dinheiro". Ele foi ouvido.

Para obter uma mobilização internacional sem precedentes, o Conselho de Segurança da ONU, pela primeira vez em sua história, inscreveu a aids na ordem do dia de suas reuniões de janeiro de 2000, que ensejariam diversas resoluções do Conselho de Segurança, da Assembleia Geral e de uma Sessão Especial da Assembleia Geral (Ungass) em 2000 e 2001 (Kerouedan, 2013). É o caso da resolução de junho de 2001 que evoca a criação de um "fundo para saúde e aids".

Apoiar o estabelecimento de um fundo mundial para o HIV/Aids e a saúde de modo a financiar uma resposta urgente à epidemia baseada em um enfoque integrado de prevenção, atenção, apoio e tratamento, e prestar assistência aos governos, entre outras coisas, em seus esforços de combate ao HIV/Aids, com a devida prioridade aos países mais afetados, notadamente da África Subsaariana e do Caribe, além de outros países em alto risco, e mobilizar as contribuições ao fundo de fontes públicas e privadas, recorrendo especialmente a países doadores, fundações, setor empresarial - inclusive a indústria farmacêutica -, setor privado, filantropos e pessoas ricas (Ungass, 2001) ${ }^{1}$.

Na prática, o fundo surgiu graças ao secretário-geral - à época, Kofi Annan, um africano sensível às repercussões humanitárias da aids sobre o continente - que obteve

\footnotetext{
${ }^{1}$ No original, "Support the establishment of a global HIV/Aids and health fund to finance an urgent response to the epidemic based on an integrated approach to prevention, care, support, treatment and to assist governments, inter alia, in their efforts to combat HIV/Aids with due priority to the most affected countries, notably in sub-Saharan Africa and the Caribbean and to those countries at high risk, and mobilise contributions to the fund from public and private sources with a special appeal to donor countries, foundations, the business community, including pharmaceutical companies, the private sector, philanthropists and wealthy individuals".
} 
junto aos Estados do G8, reunidos em Okinawa (Japão) em 2000 e em Gênova (Itália) em 2001, compromissos financeiros sem precedentes em favor de três doenças: aids, tuberculose e malária (Fundo Mundial de Luta contra Aids, Tuberculose e Malária, doravante referido como Fundo Mundial). O presidente George W. Bush, por sua vez, obteve o financiamento mais importante da história da ajuda norte-americana para a saúde internacional, porém em favor de uma só doença: a aids. O US President Emergency Program for Aids Relief (Pepfar) foi criado em 2003 para financiar a luta contra a aids em quinze países que mantinham relações estratégicas com os Estados Unidos. A Costa do Marfim foi, naquele momento, o único país francófono a ser beneficiado pelos investimentos norte-americanos. Como revela a análise da primeira fase desse programa, as intervenções foram essencialmente verticais, mais voltadas ao aumento do número de pacientes em tratamento do que à prevenção da transmissão do vírus ou o fortalecimento dos sistemas de saúde nacional (IOM, 2007; Kerouedan, 2010, 2011).

Vê-se claramente que a genealogia dos processos decisórios vinculados à criação do Fundo Mundial inspirou a Declaração do Milênio, que define os oito Objetivos de Desenvolvimento do Milênio (ODMs), dos quais três referem-se à saúde: reduzir a mortalidade infantil (ODM 4); melhorar a saúde das gestantes (ODM 5); e combater o $\mathrm{HIV} /$ Aids, a malária e outras doenças (ODM 6). Da mesma forma, esses processos decisórios precederam e inspiraram as recomendações da Comissão Macroeconomia e Saúde da OMS, presidida por Jeffrey Sachs, cujas conclusões conclamam a comunidade internacional e os países, uma vez mais em 2001, a "investir na saúde" com vistas ao financiamento da implementação dos ODMs (OMS, 2001).

O entusiasmo da mobilização em favor das citadas três doenças respaldava-se no observado há pouco tempo em 
prol da vacinação, quando se criou, em 2000, a Aliança Mundial para Vacinas e Imunização (GAVI) graças à doação de 750 milhões de dólares da Fundação Bill e Melinda Gates. Isso contagiou a fase de implementação dos ODMs de saúde, aos quais a comunidade internacional terminou por prestar uma atenção política e financeira bastante assimétrica. O ODM 5, que consistia em eliminar três quartos da mortalidade materna foi o objetivo menos financiado, a ponto de o atual secretário-geral da ONU, Ban Ki-moon, lançar uma estratégia específica para esse fim, em 2010, durante a Cúpula do G8 de Muskoka (Canadá). A situação é preocupante, pois os indicadores relativos a gravidez precoce e mortalidade de adolescentes são os que menos progrediram em vinte anos (1990-2010). Os progressos foram ainda mais lentos entre os anos 2000 e 2010 (ODI, 2012). As complicações envolvendo a gravidez de adolescentes costumam matar duas crianças (mãe e filho), 52 comprometendo a realização dos ODMs especialmente na África, onde o fenômeno é mais frequente.

De todo modo, a criação de parcerias público-privadas em escala mundial, tais como a GAVI e o Fundo Mundial mencionados, criados entre os anos 2000 e 2001, constitui uma das dimensões da emergência da "saúde global". A tradução literal da expressão "global health" do inglês para o francês corresponderia a "santé mondiale", do mesmo modo que "globalization" é em geral traduzida como "mondialisation". Nós fizemos a escolha de traduzir "global health" como "saúde mundial", o que não prejulga a interpretação feita por nossos colegas francófonos, especialmente na África, onde eles parecem tentados a falar de "santé globale", pouco importando se essa tradução veicula um conceito cujo conteúdo repercute claramente sobre o exercício e a expressão das políticas sanitárias nacionais e suas relações com os parceiros 
internacionais ${ }^{2}$. Note-se que o próprio nome da OMS foi mal traduzido: "The World Health Organisation" deveria ter sido traduzida como Organização da Saúde Mundial, pois, na expressão anglófona, é a saúde, e não a organização, que se torna mundial quando da criação da OMS no âmbito da ONU. Assim, a saúde mundial teria nascido no século XIX por ocasião das conferências internacionais sanitárias, e renascido após a Segunda Guerra Mundial.

A diversificação das modalidades de financiamento do desenvolvimento no período de 2005 a 2010 deve-se também, e talvez sobretudo, ao fato de que os Estados do Comitê de Assistência ao Desenvolvimento da Organização para a Cooperação e o Desenvolvimento Econômico (OCDE) não respeitaram seus compromissos, embora renovados em Monterrey (México) em 2002, de consagrar 0,7\% de seu produto nacional bruto (PIB) para ajuda ao desenvolvimento, tendo tornado imperativa a busca de financiamento em outras fontes.

Nesse espírito, o presidente da França à época, Jacques Chirac, encomendou em 2004 um estudo ao inspetor geral de finanças Jean-Pierre Landau, cujo relatório sugeria a criação de mecanismos inovadores de financiamento do desenvolvimento (Landau, 2004). Por iniciativa de diversos países, entre eles a França, a Unitaid ${ }^{3}$ foi criada em 2006,

\footnotetext{
2 N. da T.: No Brasil, a palavra "globalization" é traduzida como "globalização", privando de sentido a polêmica em curso na literatura francófona sobre a tradução da expressão "global health". Isto não significa que aqui não exista uma disputa de fundo sobre o conceito de saúde global (demonstrada, por exemplo, por Fortes e Ribeiro, 2014). Em respeito à autora, a tradução demarca a diferença estabelecida no artigo. Assim, quando a autora emprega a expressão "santé mondiale", ela é aqui traduzida como "saúde mundial". Quando a autora utiliza a expressão em inglês, a tradução corresponde a "saúde global", mantendo as aspas que figuram no texto original.

3 N. do E.: Além da França, integram a Unitaid os seguintes países: Brasil, Chile, Noruega e Reino Unido. A ideia dessa organização é justamente pensar e planejar novas formas de obter investimentos que previnam as três doenças em questão - aids, tuberculose e malária -, que as tratem de modo eficaz e com baixo custo, acelerando sua cura. Mais informações em: <http://www.unitaid.org/en/>.
} 
graças à cobrança de uma taxa sobre bilhetes aéreos, a fim de implementar uma forma perene de financiamento e de tranquilizar a indústria farmacêutica, pouco propensa a produzir medicamentos em grande quantidade em favor de um mercado de pobres. Aids, tuberculose e malária constituem o fulcro do mandato da Unitaid. Por outro lado, o Mecanismo de Financiamento Internacional para Imunização (IFFIm) foi instituído para financiar a GAVI (vacinas). Seguiram-se a ele outros mecanismos inovadores de financiamento, submetidos aos Estados por iniciativa da França, que se encontrava à testa de uma Task Force on Innovating Financing for Health, visando especialmente acelerar a produção e a comercialização de vacinas (Porcher e Kerouedan, 2011). Diga-se de passagem, o denominador comum desse interesse "mundial" pela saúde foi a constatação de que apenas as doenças infecciosas interessam aos investidores. Na prática, apenas os portadores de doenças infecciosas 54 ou que poderiam sê-lo tiveram (quando o tiveram) acesso a tratamentos de prevenção ou cura, bem mais do que outros pacientes. Assim, se a nova governança da saúde evoca uma "mundialidade" da saúde, ela é, na realidade, muito parcial e concerne apenas a um punhado de doenças, sobretudo de doentes. Logo, a governança da saúde mundial produziu e construiu desigualdades e iniquidades em países onde as populações já delas padeciam, tanto internamente como em relação a outros países. Trata-se de um jogo perverso que, sob o manto das boas intenções, agrava, acelera e aumenta as desigualdades e iniquidades no campo da saúde.

A despeito das disposições da Declaração de Paris sobre a Eficácia da Ajuda ao Desenvolvimento (OCDE, 2005), pelas quais países e doadores comprometem-se a alinhar-se de acordo com prioridades, e os sistemas nacionais de saúde e de gestão pública a harmonizar seus procedimentos administrativos de financiamento no plano nacional, persistiu nos países a dispersão das estruturas de gestão, abas- 
tecimento, acompanhamento e avaliação dos programas de ajuda ao desenvolvimento, ao longo de toda a implementação das iniciativas mundiais e das novas modalidades de financiamento. O setor saúde foi escolhido pela OCDE como um setor teste para exploração da governança da ajuda pública e privada e exploração da realização dos princípios da Declaração de Paris (OCDE, 2009). Essa escolha deve-se ao fato de que, por um lado, os atores, as temáticas, as estratégias e as modalidades de financiamento do setor saúde, particularmente numerosos e dispersos, prestam-se naturalmente a esse exame; e, por outro lado, porque esse setor aparenta, à primeira vista, ser a menina dos olhos dos ODMs. O setor saúde, encarado como "tracer sector" da eficácia da ajuda, torna-se ao mesmo tempo um setor piloto para estudo dos financiamentos inovadores do desenvolvimento e um revelador das modalidades de governança da ajuda ao desenvolvimento em escala mundial.

Após ter apresentado as que nos parecem ser as três grandes condições de emergência da noção de saúde mundial - (i) o risco pandêmico e outros problemas sanitários comuns; (ii) as evoluções institucionais da ajuda ao desenvolvimento em saúde; e (iii) a genealogia de uma definição comum da saúde mundial -, passamos a discutir os riscos potenciais para as populações mais pobres do planeta, trazidos pela tentação de globalização que acompanha a noção de "saúde global", com, do mesmo modo que qualquer formação totalizante, o risco totalitário que a acompanha, ainda e sempre às custas das pessoas mais frágeis, mais pobres, mais doentes, e também menos favorecidas: as mulheres e as crianças.

\section{Emergência do conceito: riscos sanitários pandêmicos, problemas comuns e soluções comuns}

Nossa pesquisa indica que a expressão "saúde global" aparece pela primeira vez em um documento do Institute of 
Medicine (IOM), instância de referência científica vinculada à Academia de Ciências dos Estados Unidos. O relatório publicado em 1997 apresenta a saúde mundial como "um interesse vital para a América" - no original, America's vital interest in global health... (IOM, 1997). O segundo capítulo do mesmo relatório, intitulado "a globalização da saúde: problemas comuns, necessidades comuns", introduz o tema da seguinte forma:

Esse capítulo mostra que as nações do mundo, incluídos os Estados Unidos, agora têm demais em comum para que a saúde seja considerada um assunto meramente nacional. Em lugar disso, um novo conceito de "saúde global" é necessário para que se enfrente problemas de saúde que transcendem as fronteiras nacionais, que podem ser influenciados por circunstâncias e experiências de outros países, e que são mais bem tratados por ações e soluções de cooperação [...] Sendo os riscos também disseminados, é o HIV a mais importante das novas infecções, tanto globalmente como nos Estados Unidos. Velhas enfermidades estão ressurgindo, como tuberculose, malária e cólera (IOM, 1997) ${ }^{4}$.

Logo, a ideia fundadora da "saúde global" seria proveniente do sentimento de que qualquer evento de saúde que se produza em determinado ponto do globo terrestre traz em si um potencial de ameaça para as populações ou para a segurança nacional de um país em outro ponto do globo. Isso se deve ao desenvolvimento espetacular e rápido dos transportes e

\footnotetext{
${ }^{4}$ No original, "This chapter shows that the world's nations, the US included, now have too much in common to consider health as a merely a national issue. Instead, a new concept of 'global health' is required to deal with health problems that transcend national boundaries, that may be influenced by circumstances and experiences in other countries, and that are best addressed by cooperative actions and solutions [...] The risks are being transferred too, HIV is by far the most important of the new infections, both globally and in the United States. Older diseases are resurging, tuberculosis, malaria and cholera".
} 
das comunicações, associados à mundialização econômica e financeira, facilitando a propagação das doenças no rastro dos homens. As características da globalização, aliás, aplicam-se muito bem à saúde: (i) a internacionalização para além das fronteiras; (ii) a liberalização facilitando as trocas que ultrapassam as restrições dos Estados para criar um mercado econômico mundial aberto; (iii) a universalização que permite o compartilhamento de experiências e de saberes em escala mundial; (iv) a desterritorialização em que a geografia importa menos do que a amplitude dos temas; e (v) a ocidentalização e a modernização de enfoques e visões de mundo.

Segundo Yach e Bettcher (1998), um ano após a publicação do relatório do IOM, o "novo paradigma" da globalização consubstancia o processo de incremento da interdependência econômica, política e social, e a integração por meio de capitais, bens, pessoas, conceitos, imagens e ideias que cruzam as fronteiras estatais.

O risco infeccioso e o risco pandêmico encontram-se no coração da ideia e da emergência do conceito de "saúde global”, mas eles não são seus únicos elementos. Um certo número de problemáticas complexas exige respostas que doravante deverão ser pensadas conjuntamente: a penúria de pessoal de saúde transforma-se em crise mundial, que revela a incipiência da realização dos ODMs; a repercussão sobre a saúde de políticas comerciais e agrícolas, ou as inter-relações entre o comércio e o princípio da propriedade intelectual, que limitam o acesso dos doentes mais pobres do mundo aos medicamentos, até mesmo os essenciais e genéricos; a repercussão das mudanças climáticas sobre a saúde; as migrações e a saúde etc. As doenças crônicas não mais correspondem a perfis patológicos relativos apenas aos países ricos, mas a uma urbanização galopante que se produz também nos países pobres e emergentes. Note-se que, embora o envelhecimento das populações e os perfis patológicos tendam a uniformizar-se entre os hemisférios Norte e o Sul, com as doenças crônicas 
tornando-se as maiores causas de mortalidade em escala mundial, inclusive nos países emergentes, não são esses os fenômenos que caracterizam a saúde mundial. Eles só foram levados em consideração quinze anos após a publicação do relatório do IOM. Somente em setembro de 2011 é que as doenças crônicas entraram na ordem do dia da Assembleia Geral das Nações Unidas, dez anos após a inclusão da aids na pauta do Conselho de Segurança.

\section{Evoluções institucionais: a governança mundial da saúde}

Abordando a "globalização da saúde internacional", Gill Walt (1998) demonstra como a globalização se aplica à saúde no campo internacional, engendrando novas coalizões e alianças: "com o fortalecimento da globalização do comércio e dos mercados, novas coalizões e alianças formam-se para avaliar e enfrentar suas consequências diretas e indiretas sobre a saúde".

A evolução institucional das modalidades de financiamento da ajuda ao desenvolvimento em favor da saúde marcou significativamente as representações da "saúde global", especialmente nos países receptores desses financiamentos. Os atores dos países beneficiários de fundos mundiais foram convidados a implementar novas instâncias nacionais, tais como o Country Coordinating Mechanism (CCM) do Fundo Mundial. Trata-se de uma instância de concepção e direção de projetos financiados por esse fundo e que reúne atores públicos, privados e comunitários; chamam-no de CCM inclusive nos países francófonos africanos. Assim, a "saúde global”, como a denominam os atores locais, é percebida por esses países como estreitamente vinculada às instâncias de governança de iniciativas mundiais, em particular do Fundo Mundial, ou melhor, do Global Fund, citado em inglês mesmo nos países francófonos. Aqui, a noção de "saúde global" presta-se por vezes à confusão com uma expressão mais antiga de abordagem "global" das doenças, amiúde referente à 
assistência às pessoas com aids, convidando os profissionais da saúde a considerar simultaneamente as dimensões psicológica, diagnóstica e terapêutica da doença. Por outro lado, a genealogia e a emergência da noção de saúde mundial são independentes e distintas da noção de bem público mundial, cuja expressão foi muito utilizada no âmbito das políticas de desenvolvimento do início da década de 2000.

Os nomes das estratégias internacionais das instituições de cooperação sanitária bilateral, como o Reino Unido e a Comissão Europeia, inspiram-se no mesmo vocabulário das iniciativas mundiais que se referem à saúde mundial como "global". Nesse sentido, a Comissão Europeia publicou, em 2010, uma Comunicação ao Conselho da União Europeia e ao Parlamento Europeu intitulada "EU role in global health" (Comissão Europeia, 2010).

\section{Rumo a uma definição comum da "saúde global"}

Diante das diversas interpretações da noção de "saúde global", uma definição consensual foi proposta por um grupo de pesquisadores:

Saúde global é uma área de estudo, pesquisa e prática que dá prioridade à melhora da saúde e à conquista da equidade em saúde para todos os povos do mundo. A saúde global enfatiza questões, determinantes e soluções transnacionais de saúde; envolve muitas disciplinas, inclusive para além das ciências da saúde, promovendo a colaboração interdisciplinar; e é uma síntese entre a prevenção de base populacional e o atendimento clínico no plano individual (Koplan et al., 2009) ${ }^{5}$.

\footnotetext{
${ }^{5}$ No original, "Global health is an area for study, research and practice that places a priority on improving health and achieving equity in health for all people worldwide. Global health emphasizes transnational health issues, determinants and solutions; involves many disciplines within and beyond the health sciences and promotes interdisciplinary collaboration; and is a synthesis of population-based prevention with individual-level clinical care".
} 
Essa definição insiste no fato de que cada pessoa do planeta tem direito à boa saúde, sendo a equidade um valor filosófico fundamental da saúde pública. "A promoção da equidade social e econômica e a redução das desigualdades em matéria de saúde tornaram-se um tema chave da saúde pública interna, da saúde internacional e da saúde global" (Koplan et al., 2009).

Sem se referir ao documento publicado em 1997 pelo IOM, que mencionou pela primeira vez a ideia de "saúde global”, provavelmente desconhecido dos autores, Koplan e seus colaboradores convidam a uma definição comum dessa expressão a partir de uma concepção genealógica do conceito, inscrevendo-o na continuidade das noções de saúde pública e de saúde internacional. Eles apresentam uma tabela didática comparativa entre saúde pública, "international health" e "saúde global". Os valores, os princípios e os métodos são comuns aos três campos. A seguir, 60 enunciam suas características a partir das diferenças entre eles: enquanto a saúde pública se interessaria pela saúde das populações no plano nacional, a "international health" diria respeito à geografia dos países em desenvolvimento, o que foi chamado em francês, por quarenta anos, de "saúde e desenvolvimento" ("santé et développement"). A "saúde global”, por sua vez, faria referência à amplitude dos problemas e não à sua localização geográfica (Koplan et al., 2009), sendo todo e cada ser humano a população-alvo da "saúde global". A localização geográfica dos países em desenvolvimento não mais aparecerá na definição de saúde mundial. Nas palavras de Ilona Kickbusch (2009), o desafio da saúde mundial é reconciliar os enfoques globais da saúde com aqueles que se interessam especialmente pelos países em desenvolvimento. Koplan e seus colegas insistem sobre o fato de que, embora seja importante ter uma boa compreensão da evolução da governança em escala mundial, as melhoras serão de fato provenientes da esfera local. 
Nesse sentido, afirmam: "embora a compreensão das tendências mundiais e das mudanças políticas seja importante, os avanços em matéria de saúde dependem do que ocorre nos níveis nacional, subnacional e local, e, em última instância, nas comunidades em que as famílias vivem e morrem” (Koplan et al., 2009).

Assim, vemos que a compreensão da noção de "saúde global" merece ser explicitada, tanto no âmbito dos intercâmbios internacionais como junto aos profissionais e pesquisadores que trabalham em campo. Essa expressão recobre, na realidade, interpretações que variam conforme nosso grau de interesse pelos efeitos da globalização, pelas evoluções institucionais recentes da arquitetura da ajuda mundial, ou por este valor que supostamente reúne os atores e beneficiários da saúde pública: a equidade. Se nós insistimos em um necessário esclarecimento da noção é porque isso tornaria mais fácil sua apropriação pelos atores e pesquisadores, favorecendo um diálogo e uma cooperação mais ricos de sentido, que pode coincidir com maior eficácia da ajuda ao desenvolvimento.

Também é necessário esclarecer o conteúdo dessa noção a fim de chegarmos a um entendimento quanto aos saberes e às qualificações profissionais que poderão ser requeridos e reconhecidos nas universidades e no mundo profissional: o que seria um diploma em saúde mundial? O que seria um especialista ou um profissional da saúde mundial? A Associação das Escolas de Saúde Pública americana é a única instituição que propõe um Global Health Competency Model voltado ao meio acadêmico (ASPH, 2011). Do lado europeu, a Associação de Escolas de Saúde Pública propõe um conteúdo harmonizado das competências em "saúde pública” (Aspher, 2010, 2012), e em breve fará o mesmo nos cursos relativos à "saúde global" (Haines, 2011). Em Paris, a Escola de Negócios Internacionais do Instituto de Estudos Políticos de Paris (Sciences Po, 2016) propõe 
uma especialização intitulada Concentration in Global Health, que é oferecida a estudantes matriculados em uma dezena de masters internacionais. Ao formar estudantes franceses, europeus e de outros países a respeito desses temas, o objetivo é preparar os futuros líderes políticos mundiais em todas as disciplinas para chamar para si, politicamente, as questões de saúde no âmbito de suas responsabilidades.

Já tratamos dos efeitos da nova governança mundial de saúde sobre a realidade em outras publicações, tanto em termos de governança como de eficácia da ajuda aos sistemas de saúde (Yu et al., 2008; Balabanova et al., 2010; Kerouedan, 2010; Kerouedan e Brunet-Jailly, 2011; McCoy et al., 2012). No presente texto, insistiremos sobretudo nos riscos que essa nova governança mundial da saúde traz para os pobres.

\section{Riscos da globalização da saúde para as populações mais pobres da África}

62 Ao abordar a emergência da noção de saúde mundial, constatamos que sua ideia básica é a de que seremos todos igualmente atingidos pela superveniência de um evento sanitário em qualquer ponto do globo, e de que problemas comuns complexos exigem soluções que doravante devem ser pensadas em conjunto. Esse enfoque não é desprovido de riscos, pois ele poderia ensejar a tentação de uniformizar as análises, os problemas, os enfoques e as soluções examinadas no âmbito dessa saúde "totalizada" dita "mundial". Ao globalizar assim os problemas e as soluções, o risco é de ver apenas as prioridades comuns aos países do planeta serem consideradas prioridades estratégicas internacionais, o que privaria as populações que vivem em contextos diferentes (pobres e em situação de conflitos armados, por exemplo) de conhecimentos e de respostas específicas para a diversidade de suas situações e realidade. É o que observamos no período de realização dos ODMs de saúde, de 2000 a 2015, quando a comunidade internacional privilegiou a luta contra 
a aids em detrimento das prioridades dos países mais pobres, onde a aids não constitui uma prioridade de saúde pública - por exemplo, onde a mortalidade mais elevada não é a aids, mas de mulheres grávidas ou crianças.

A tentação de padronizar análises e respostas é ainda maior quando os perfis epidemiológicos, sanitários e sociais dos países emergentes (doenças crônicas e proteção social, por exemplo) são cada vez mais assimilados aos dos países ricos, enquanto o maior número de pessoas pobres que sofrem outros problemas de saúde reside nesses países e as desigualdades sociais são vultosas, assim como as de saúde que dela decorrem. Em qualquer caso, a motivação dos países ricos para intervir é sempre a mesma: ou os problemas são compartilhados, ou não são compartilhados, mas constituem ameaças para a segurança nacional e internacional.

Assim, contrariamente às afirmações de quem a promove, e sob o pretexto de um valor filosófico compartilhado, que destaca a busca da equidade, os que tomam decisões no âmbito da saúde mundial não parecem em realidade atingidos pelo estado de saúde de toda e qualquer pessoa desse mundo, ou pelo que poderia ameaçar o acesso ao tratamento de cada uma das pessoas que integram esse "todo". Eles estariam mais tentados a concentrar sua atenção política e seus recursos apenas em direção aos problemas comuns ou considerados mais estratégicos.

O risco é, então, de se interessar pelas situações sanitárias particulares das populações pobres apenas na medida em que elas possam constituir uma ameaça potencial para a saúde ou a economia dos países mais ricos (riscos pandêmicos, penúria de pessoal médico, perdas comerciais, repercussões econômicas etc.). Ademais, essa atitude é paradoxal quando a preocupação maior é, por exemplo, de conter a propagação de uma nova pandemia: como os deficientes sistemas de saúde dos países pobres, pouco contemplados pela ajuda mundial, poderiam dispor de 
sistemas eficientes de alerta e vigilância epidemiológica, formar pessoal de saúde, implementar mecanismos perenes de financiamento do setor etc., além de outras dimensões de sistemas sustentáveis necessários à proteção da saúde de todos no mundo?

A mundialização causou mudanças: nós passamos de uma clivagem Norte-Sul a uma acentuada clivagem entre ricos e pobres, podendo os últimos estarem nos países do Norte, nos países emergentes ou nos países menos avançados (PMA). Se a globalização da saúde se faz acompanhar do financiamento prioritário pelos países ricos daquilo que eles percebem como uma ameaça para eles, independentemente da mensuração objetiva da carga de doença, corre-se o risco de ver as prioridades e as populações dos países mais pobres, onde quer que estejam, saírem do campo da saúde mundial, e também do campo do desenvolvimento. É preciso que as situações específicas dos 64 PMA e dos Estados frágeis, justamente os que apresentam maior atraso na realização dos ODMs, continuem na mira daqueles que tomam as decisões relativas aos objetivos de desenvolvimento pós-2015 (Guillaumont, 2013a, 2013b; Guillaumont e Wagner, 2013). Nos vinte anos que se seguiram à Cúpula da Terra de 1992 (ECO-92), os defensores do desenvolvimento sustentável não parecem ter atribuído à saúde o interesse que ela merece. Dos três pilares que supostamente compunham uma estratégia de desenvolvimento sustentável, apenas o pilar do meio ambiente reteve a atenção de seus profissionais e defensores, em detrimento de preocupações sociais e de equidade.

É interessante perceber que o objetivo de Cobertura Sanitária Universal (CSU) proposto pela OMS para financiar os sistemas de saúde (OMS, 2010a) parecia alcançar o consenso por ocasião dos trabalhos preparatórios da agenda do desenvolvimento pós-2015 (Haines et al., 2012). Esse objetivo pode parecer à primeira vista sedutor, mas o 
efeito que se espera dele não é tanto o de reduzir o número de pobres, mas sim de diminuir o contingente de pessoas que cai na pobreza devido aos gastos com tratamentos de saúde. O risco de escolher esse objetivo de CSU é o de continuar a levar os países em desenvolvimento a se focalizarem na questão de como financiar o tratamento dos doentes cada vez mais numerosos, em lugar de refletirem sobre as estratégias que contribuam para prevenir as doenças. São temas sobre os quais o Prêmio Nobel de Economia Amartya Sen (2000) convida nossas democracias a discutir, o que não é fácil em nossas sociedades sob o império de poderosos lobbies.

Em 2009, a Comissão sobre os Determinantes Sociais da Saúde da OMS produziu um relatório no qual lamenta: “a injustiça social é responsável pela morte de pessoas em grande escala. As desigualdades de saúde matam em grande escala", propondo que os países organizem suas políticas sanitárias a partir dos principais determinantes sociais da saúde de suas respectivas sociedades (OMS, 2010b). Assim preconiza, por exemplo, o Canadá:

[...] os principais fatores que possuem incidência sobre a saúde da população canadense nada têm a ver com os tratamentos médicos ou com as escolhas de estilo de vida, mas têm tudo a ver com as condições de vida da população. São estas últimas que formam hoje o que passou a ser chamado de determinantes sociais da saúde. Sua importância para a saúde foi primeiramente apontada em meados do século XIX, e posteriormente essa noção inscreveu-se nos documentos de política governamental em meados dos anos 1970. De fato, as contribuições de origem canadense ao conceito de determinantes sociais da saúde são tão vastas que o meio da saúde, em escala mundial, considera o Canadá uma grande força motriz em matéria de promoção da saúde. Os recentes relatórios elaborados 
pelo Administrador Chefe da Saúde Pública, do Senado e da Agência de Saúde Pública do Canadá continuam a sustentar a importância dos determinantes sociais da saúde (Mikkonen e Raphael, 2011).

Note-se que os mecanismos sobre os quais a CSU repousa, por exemplo, na França, são deficitárias a longo prazo, o que nós não gostaríamos de ver encorajado, nem mesmo nos países emergentes. O sistema de saúde é verdadeiramente um exemplo na França, no qual, de um lado, os lobbies industriais, que atuam nos gabinetes ministeriais, intoxicam as populações com seus produtos que causam dependência (medicamentos, alimentos, tabaco, álcool), e, de outro, o sistema de seguro saúde financia $100 \%$ do tratamento das doenças crônicas (por exemplo, o câncer causado por esses produtos tóxicos).

Considerando as capacidades institucionais e técnicas 66 dos países mais pobres, eles não parecem preparados para implementar tais sistemas. As políticas de saúde mundial, tal como elas se realizaram ao longo da década passada, não provaram sua eficácia para reforçar as capacidades institucionais dos países. Por todas essas razões, o risco de adoção desse objetivo de CSU, que tem pouca chance de realizar-se concretamente em benefício das populações mais pobres, é de ver acentuadas as desigualdades entre países ricos e países emergentes, de um lado, e de países pobres, do outro, mas também no seio de cada país das diversas regiões do mundo, tendo por consequência continuar não considerando as prioridades de saúde específicas das populações pobres (doenças infecciosas, mortes infantis, maternas e especialmente de adolescentes), em particular das mulheres, cujo enfrentamento exige sistemas de saúde sólidos em favor dos quais os financiamentos continuarão a faltar. 


\section{0 risco de ver novos atores determinarem a política sanitária}

A diversificação dos atores no campo da saúde internacional não é nova. A Fundação Rockefeller desempenhou um papel considerável ao longo de todo o século XX para formar especialistas e financiar as políticas sanitárias norte-americanas e internacionais, inclusive na França (APHA, 2003; Kerouedan, 2013). O que é novo na governança mundial da saúde do século XXI é a onipresença do setor privado industrial e comercial (não somente a indústria farmacêutica, mas também as fundações filantrópicas), chamadas num primeiro momento a contribuir com o financiamento de políticas públicas como parceiras. Nesse intervalo, o parceiro tornou-se decision-maker e policy-maker, conquistou poder a ponto de os escritórios de estratégia da OMS, em Genebra, terem recrutado conselheiros de Bill Gates. Se a saúde mundial passou a ser governada por Bill Gates, então não há chance alguma de que ela seja global: sua filantropia não se irradia além de algumas patologias infecciosas. Nós poderíamos seriamente qualificar de filantropia, isto é, "amor dos homens", uma ação que ama apenas alguns, e somente aqueles que são percebidos como ameaças para os outros, e preferencialmente para os mais ricos do planeta?

Outra inovação concerne ao volume de financiamento mobilizado pelas fundações privadas e pelo governo norte-americanos que, por sua importância, ditam, de fato, a política sanitária mundial, tanto por meio da ajuda bilateral pública e privada, como pelas parcerias público-privadas mundiais que eles financiam e os conselhos de administração nos quais eles passam a decidir as diretrizes e as prioridades, independentemente das prioridades epidemiológicas e sanitárias dos países. Esses desequilíbrios foram denunciados ao Tribunal de Contas Europeu (TCE, 2009; Kerouedan, 2009). Essas parcerias público- 
-privadas mundiais afirmam ser instituições financeiras e se supõe que elas assim permanecerão na paisagem institucional mundial da ajuda ao desenvolvimento. Teoricamente, compete apenas aos países e aos seus parceiros técnicos, essencialmente a ONU, escolher as estratégias sanitárias pertinentes que serão submetidas às iniciativas mundiais. Na prática, nós observamos o inverso em toda a sua perversidade: são as decisões de financiamento que ditam as políticas, e quanto maiores são os volumes de financiamento, maior é o efeito de distorção sobre os sistemas de saúde.

O Conselho de Administração do Fundo Mundial pode decidir, por exemplo, financiar prioritariamente o tratamento de pacientes com aids ou a luta contra a aids junto a certos grupos considerados "de risco". O conjunto de países se alinhará conforme essas incitações, mesmo se essas prioridades não forem as suas. Por força das priori68 dades que ela escolhe, a Fundação Bill e Melinda Gates orienta também as escolhas dos países, erodindo a sua soberania. De uma parte, a disponibilidade e o montante dos financiamentos, mais do que as necessidades, são o que define as intervenções e os programas. De outra parte, a preocupação não é o resultado sanitário, mas o resultado financeiro e a gestão dos fundos (Kerouedan, 2015). Graças aos volumes de financiamento mobilizados, as iniciativas mundiais e as fundações privadas norte-americanas adquirem uma legitimidade e uma liderança que fragilizam as da OMS, o que é discutido por numerosos autores (Brown et al., 2006, Fidler, 2010, Killen e Rogerson, 2010, Hill, 2011, Sridhar et al., 2012, Bollyky, 2012, Horton, 2012, OMS, 2013, Clift, 2013). Mais do que uma fragilização, trata-se de uma apropriação da liderança alheia, da qual os poderes públicos participam.

Quando se refere aos sistemas-mundo e às economias-mundo, Immanuel Wallerstein (2009) indica que o obje- 
tivo do hífen é "indicar que nós não falamos de sistemas, economias ou impérios referentes ao mundo inteiro, mas de sistemas, economias ou impérios que constituem um mundo - que, em geral, não inclui o mundo inteiro". Parece que passamos, na verdade, de um sistema de economia-mundo a um sistema de finanças-mundo, em que os valores das finanças e do lucro orquestram os vetores do mundo, inclusive no campo da saúde, interpretado tradicionalmente como concernente mais à caridade do que aos negócios. Estávamos habituados às metáforas da saúde pública tingidas pela ideia de solidariedade, de preocupações comerciais ou mesmo de interesses diplomáticos ou securitários. O campo é agora ocupado por lógicas financeiras (Kerouedan, 2014).

Esses fenômenos observados no campo da saúde dizem muito sobre fenômenos globais que estão em curso, entre eles a despolitização e a marginalização dos poderes públicos, inclusive Estados, por ação do setor privado (Livinec e Kerouedan, 2015). O liberalismo alcançou assim sua imanência mais total, até tomar para si todas as missões de serviço público, inclusive ser prestatário das missões mais indissociáveis da soberania do Estado, como a defesa nacional. Não podemos excluir a hipótese de que essa despolitização, essa desapropriação das missões do Estado que são sistematicamente contornadas pelos mecanismos da ajuda mundial no campo da saúde, tenha contribuído de forma evidente à fragilizacão dos Estados africanos, já institucionalmente frágeis, e ao seu descrédito nacional, aos olhos de seus próprios povos. Assim, o liberalismo sem limites pode ter favorecido a emergência de grupos extremistas que vieram instalar-se no vazio estatal, num primeiro momento nas regiões geográficas mais recuadas do Sahel, mas atualmente cidades e o coração dos centros das capitais já foram atingidos, como Bamako (Mali) e Ouagadougou (Burquina Faso). 


\section{0 risco de tomar decisões políticas sem conhecer a realidade local}

Não sendo representadas nos Estados, como são, por exemplo, as agências das Nações Unidas ou as instituições de ajuda bilateral, as instâncias dirigentes executivas ou políticas das parcerias público-privadas mundiais (como o Fundo Global e a GAVI) tendem a emitir decisões sem levar em consideração as realidades e condicionantes locais, cujas dinâmicas e transformações nunca foram tão rápidas. Dirigentes atarefados privam-se frequentemente dos saberes e do conhecimento dos atores de campo, e das trocas intelectuais com pesquisadores, atores políticos, sociedade civil e universitários que se encontram nesses locais. Os saberes produzidos nem sempre são acessíveis aos que tomam as decisões políticas. Esse distanciamento do real pode constituir uma das principais causas, embora pouco comentadas, da limitada eficácia da ajuda financeira. Ainda que esta

70 tenha crescido, a avaliação de sua performance privilegia a escolha de indicadores quantitativos.

À guisa de conclusão, a emergência da noção, senão do conceito, de "saúde global" permite às comunidades científica e profissional recordar os princípios da saúde pública, entre eles o valor filosófico fundamental da equidade. Observações feitas em campo ao longo dos dez últimos anos, em especial quando da avaliação dos cinco anos do Fundo Mundial, permitiram constatar uma deriva da saúde mundial, em contradição com o princípio de equidade, na qual o paciente com aids, por exemplo, poderá beneficiar-se de medicamentos gratuitos no mesmo hospital em que um paciente com qualquer outra doença ou uma jovem gestante cujo parto se complica podem morrer em plena indiferença (Macro International, 2009; Kerouedan, 2010). Há aqui uma questão ética pouco abordada pelos dirigentes das instâncias públicas e privadas que escolhem, na prática, as pessoas e os pacientes que podem ser salvos, 
conforme as ameaças e os riscos que esses pacientes representam (ou não) para as populações e a segurança das nações ricas - é o que se pode deduzir dos relatórios do Conselho Nacional de Inteligência dos Estados Unidos (NIC, 2000, 2002, 2003, 2008).

As autoridades sanitárias legítimas estão sobrecarregadas e não conseguem desempenhar o seu papel científico, técnico e regulador de fazer com que os financiadores públicos ou privados baseiem a alocação da ajuda em critérios objetivos, como a carga de morbidade ou de mortalidade, a população, a pobreza etc., em lugar de interesses geopolíticos, econômicos ou comerciais. O Institute for Health Metrics and Evaluation demonstra a desconexão que ocorre há anos entre a carga de doença e a repartição da ajuda mundial à saúde (IHME, 2013) .

Recordemos que os médicos e diplomatas reunidos em Paris, em 1851, à mesa da primeira conferência sanitária internacional, reunião que funda sem dúvida a ideia de saúde mundial, foram confrontados às mesmas tensões entre interesses comerciais e proteção sanitária que a medida de quarentena, à época, buscava impor (Kerouedan, 2013). Todo o desafio é então, e cabe a todos compartilhar essa responsabilidade, o de reduzir ao mínimo esse paradoxo inerente à saúde mundial, em favor do desenvolvimento do potencial de equidade e de redução das desigualdades. A precariedade da situação na África, especialmente a francófona, merece toda a nossa atenção, sob pena de ver as instabilidades políticas e a pobreza alimentarem os conflitos (Kerouedan, 2012), o que já observamos largamente.

Assim, encontramos aqui um grande paradoxo da saúde mundial, cuja gênese encontra-se precisamente numa questão de saúde percebida à época como assunto de segurança nacional e internacional: de tanto querer se ocupar da segurança mundial em termos sanitários, os atores ditos filantrópicos talvez possam, de forma muito insidiosa mas 
bastante concreta, contribuir também com a fragilização das entidades estatais dos países pobres, favorecendo assim a insegurança nacional e internacional, cuja preservação eles teriam adotado como objetivo.

\section{Dominique Kerouedan}

é professora da Paris School of International Affairs (PSIA - Sciences Po Paris), cátedra Geopolítica da Saúde Mundial do Collège de France (2012-2013).

\section{Bibliografia}

ACSI - Aids, Security and Conflict Initiative. 2010. VIH/sida, sécurité et conflits: nouvelles réalités, nouvelles réponses. Revue Migrations Forcées, Supplément Spécial: VIH/sida, sécurité et conflits: établir les liens. Disponível em: <http://www.fmreview.org/sites/fmr/files/ FMRdownloads/fr/SIDA.pdf $>$. Acesso em: 10 jul. 2016.

APHA - American Public Health Association. 2003. Growth of international health. An analysis and history. Washington, DC: APHA. ASPH - The Association of Schools of Public Health. 2011. Global Competency Model. Disponível em: <https://www.publichealth.pitt.edu/Portals/0/ Main/ASPH\%20GH\%20Competencies.pdf>. Acesso em: 11 jul. 2016. ASPHER - The Association of Schools of Public Health in the European Region. 2010. Provisional lists of public health core competencies. European Public Health Core Competencies. Phases 1 and 2. Bruxelas: Aspher. 2012. Aspher's position paper concerning: the new European Policy for health - Health 2020 (Draft2). Bruxelas: Aspher.

BALABANOVA, D. et al. 2010. What can global health institutions do to help strengthen health systems in low income countries?. Health Research Policy and Systems, n. 8, p. 22.

BALIQUE, H. 2011. Le défi de la santé en Afrique subsaharienne et ses perspectives. In: KEROUEDAN, D. (dir.). Santé internationale: les enjeux de santé au Sud. Paris: Presses de Sciences Po.

BANCO MUNDIAL. 1993. Investir dans la santé. Washington, DC: Banco Mundial.

BOLLYKY, T. 2012. Reinventing the World Health Organization. New York/ Washington, DC: Council on Foreign Relations.

BROWN, T. et al. 2006. The World Health Organization and the transition from international to global public health. American Journal of Public Health, v. 96, n. 1. 
CLIFT, C. 2013. The role of the World Health Organization in the International System. Centre on Global Health Security. Working group on governance. Working Group Papers, n. 1. Disponível em: <https://www.chathamhouse.org/sites/files/chathamhouse/public/ Research/Global\%20Health/0213_who.pdf>. Acesso em: 12 jul. 2016. COMISSÃO EUROPEIA. 2010. Le rôle de l'UE dans la santé mondiale. Communication de la Commission Européenne au Conseil et au Parlement Européen, au Comité Économique et Social Européen et au Comité des Régions. Bruxelas. Disponível em: <http:/ / ec.europa.eu/ europeaid/sites/devco/files/communication-eu-role-in-global-healthcom2010128-20100331_fr.pdf>. Acesso em: 11 jul. 2016.

FIDLER, D. P. 2010. The challenges for global health governance. New York/ Washington, DC: Council on Foreign Relations. Disponível em: <http:/ /www.cfr.org/global-governance/challenges-global-healthgovernance/p22202>. Acesso em: 13 jul. 2016.

FORTES, P.; RIBEIRO, H. 2014. Saúde global em tempos de globalização. Saúde e Sociedade, v. 23, n. 2, pp. 366-75.

GUILLAUMONT, P. 2013a. Quels objectifs pour le développement après 2015? Point d'étape et intégration de la durabilité environnementale. Communication à la Conférence conjointe du Ferdi-IDDRI-MAE. Paris. . 2013b. Les pays vulnérables dans l'agenda post 2015. Ferdi Note Breve, n. B66.

GUILLAUMONT, P.; WAGNER, L. 2013. Measuring structural vulnerability to allocate development assistance and adaptation resources. Development Policies Working Paper. Ferdi, n. 68.

HAINES, A. et al. 2011. European academic institutions for global health. The Lancet, n. 377, pp. 363-65.

2012. From the Earth Summit to Rio +20: integration of health and sustainable development. The Lancet, n. 379, pp. 2189-97.

HILL, P. 2011. Understanding global health governance as a complex adaptative system. Global Public Health, v. 6, n. 6, pp. 593-605.

HORTON, R. 2012. "Offline": Global health-an end of term report. The Lancet, n. 379, p. 1934.

IHME - Institute for Health Metrics and Evaluation. 2013. Financing global health 2012: The end of the Golden Age? Seattle: IHME.

IOM - Institute of Medicine. 1997. America's vital interest in global health: protecting our people, enhancing our economy, and advancing our international interests. Washington, DC: IOM. 2007. Pepfar Implementation. Progress and promise. Washington, DC: IOM. 
KEROUEDAN, D. 2009. Health and development financing. The Lancet, n. 374, pp. $435-36$.

2010. Les enjeux politiques de l'évaluation à cinq ans du Fonds Mondial de lutte contre le sida, la tuberculose et le paludisme. Médecine Tropicale, n. 70, pp. 19-27.

. 2011. Evolutions de l'architecture internationale de l'aide en faveur de la santé dans les pays en développement. In: KEROUEDAN, D. (dir.). Santé internationale: les enjeux de santé au Sud. Paris: Presses de Sciences Po.

. 2012. La santé en Afrique: pourquoi la France et l'Europe devraient être plus engagés. In: MENASCE, D. (dir.). Lutte contre la pauvreté, entre don et marché. FACTS Reports. Disponível em: <https:/ / factsreports.revues.org/1238>. Acesso em: 13 jul. 2016.

2013. Géopolitique de la santé mondiale. Conférence inaugurale au Collège de France. Paris: Fayard.

. 2014. La vocation altruiste dans un monde globalisé. Conférence inaugurale du Colloque International de Philosophie Politique Survie, Vie digne, Qualité de vie, dans les situations extrêmes d'inégalisation globale. Disponível em: <https://www.youtube.com/watch?v=U0k4cv_ fen8>.

2015. Les bonnes pratiques de la "global health": améliorer la santé ou bien gérer l'argent?, In: KLEIN, A.; LAPORTE, C.; MARIE, C. (dirs.). Les bonnes pratiques des organisations internationales. Paris: Presses de Sciences Po.

KEROUEDAN, D.; Brunet-Jailly, J. 2011. Analyse de la pertinence et de l'efficience des financements du Fonds Mondial en appui à la prévention du VIH/Sida en Côte d'Ivoire. Rapport à l'Onusida. [s.l.].

KICKBUSH, I. 2009. Prof. Ilona Kickbusch presents the Global Health Programme. Vídeo. Genebra: The Graduate Institute. Disponível em: $<$ https://www. youtube.com/watch?v=6tPkt-ucnnc $>$. Acesso em: 4 mar. 2016.

KILLEN, B.; ROGERSON, A. 2010. Global governance for international development: who is in charge?. Developement Brief, OCDE, n. 2.

KOPLAN, J. et al. 2009. Towards a common definition of global health", The Lancet, n. 373, pp. 1993-95.

LANDAU, J.-P. 2004. Les nouvelles contributions financières internationales: financement et développement et taxations internationales. Rapport au Président de la République. Paris: La Documentation Française (Collection des rapports officiels). Disponível em: <http://www. ladocumentationfrancaise.fr/var/storage/rapports-publics/044000440. pdf $>$. Acesso em: 11 jul. 2016. 
LIVINEC, B.; KEROUEDAN, D. 2015. Philanthrocapitalisme et santé, une générosité au service de la géopolitique néolibérale?. Les Convivialistes. Disponível em: <http://www.lesconvivialistes.org/textes-et-debats/ debats/175-philanthrocapitalisme-en-sante-une-generosite-au-servicede-la-geopolitique-neoliberale-par-bertrand-livinec-et-dominiquekerouedan>. Acesso em: 11 jul. 2016.

MACRO INTERNATIONAL. 2009. Global Fund 5-y Evaluation. The impact of collective efforts on the reduction of diseases burden. Disponível em: $<$ www.theglobalfund.org $>$.

MIKKONEN, J.; RAPHAEL, D. 2011. Déterminants sociaux de la santé: les réalités canadiennes. Toronto: École de Gestion et de Politique de la Santé de l'Université York.

MCCOY, D. et al. 2012. The Global Fund: what next for aid effectiveness and health systems strengthening? Nairobi (Quênia): Aidspan.

NIC - National Intelligence Council. 2000. The global infectious diseases threats and its implications for the United States.

2002. The next wave of HIV/Aids: Nigeria, Ethiopia, Russia, India, and China.

2003. SARS: down but still a threat.

2008. Strategic implications of global health.

OCDE - Organização para a Cooperação e o Desenvolvimento

Econômico. 2005. Déclaration de Paris sur l'efficacité de l'aide et

l'harmonisation. [et Programme d'action d'Accra (2008)]. Disponível em: <https://www.oecd.org/fr/cad/efficacite/34579826.pdf>. Acesso em: 11 jul. 2016.

2009. Aid for better health, what are we learning about what works and what we still have to do? An Interim report from the Task Team on Health as a tracer sector. Working Party on Aid Effectiveness. Paris: OECD-DAC. ODI - Overseas Development Institute. Save the Children. 2012. Charting the future. Empowering girls to prevent early pregnancy. [by Elizabeth PreslerMarshall and Nicola Jones]. Disponível em: <https://www.odi.org/ sites/odi.org.uk/files/odi-assets/publications-opinion-files/7724.pdf>. Acesso em: 11 jul. 2016.

OMS - Organização Mundial da Saúde. 2001. Commission

Macroéconomie et Santé. Présidée par J. Sachs.

. 2010a. Le financement des systèmes de santé: le chemin vers la couverture universelle. Rapport sur la santé dans le monde. Genebra: OMS.

2010b. Redução das desigualdades no período de uma geração. Igualdade na saúde através da acção sobre os seus determinantes sociais. Relatório Final da Comissão para os Determinantes Sociais da Saúde. Lisboa: OMS. 
2013. WHO's role in global health governance. Report by the director general. Executive board, Jan. 18.

ONU - Organização das Nações Unidas. 2012. The millennium development goals report. New York: ONU.

PIOT, P. 2011. Résumé de cours. Chaire Savoirs contre pauvreté 2009-2010.

Collège de France. Chaires annuelles. Disponível em: <www.college-defrance.fr $>$. Acesso em: 10 jul. 2016.

PORCHER, D.; KEROUEDAN, D. 2011. Health financing evolving context, evolving methods. A review of innovating financing for health. Ministère [francês] des affaires étrangères. Disponível em: <http://docplayer. net/2371228-Health-financing-evolving-context-evolving-methods-areview-on-innovative-financing-for-health.html>. Acesso em: 11 jul. 2016.

SCIENCES PO. 2016. Global health concentration - Why offer training on global health at Sciences Po? Disponível em: <http:/ / www.sciencespo.fr/psia/ content/global-health-concentration>. Acesso em: 4 mar. 2016.

SEN, A. 2000. Santé et développement. Bulletin de l'OMS, recueil d'articles, n. 2. SRIDHAR, D. et al. 2012. Health governance. How the WHO can regain its relevance. Foreign Affairs. Disponível em <https://www.foreignaffairs. com/articles/2012-05-24/healthy-governance>. Acesso em: 4 mar. 2016.

TCE - Tribunal de Contas Europeu. 2009. EC development assistance to health services in Sub Saharan Africa. Special Report 10, 2008. Luxemburgo: TCE.

UNAIDS - Joint United Nations Program on HIV/Aids [Programa Conjunto das Nações Unidas sobre HIV/Aids]. 2013. Global report: Unaids report on the global aids epidemic 2013. Disponível em: <http:/ / files.unaids.org/en/media/unaids/contentassets/documents/ epidemiology/2013/gr2013/UNAIDS_Global_Report_2013_en.pdf>. Acesso em: 10 jul. 2016.

UNGASS - Sessão Especial da Assembleia Geral [da ONU]. 2001. Déclaration d'engagement pour la lutte contre le VIH/sida. Résolution de la session spéciale de l'Assemblée Générale des Nations Unies, June 27.

WALLERSTEIN, I. 2009. Comprendre le monde. Introduction à l'analyse des systèmes-monde. Paris: La Découverte.

WALT, G. 1998. Globalisation of international health. The Lancet, n. 351, pp. 434-37.

YACH, D.; BETTCHER, D. 1998. The globalisation of public health, II: the convergence of self-interest and altruism. Public health Policy Forum, v. 88, n. 5, pp. 738-41.

YU, D. et al. 2008. Investment in HIV/Aids programs: does it help strengthen health systems in developing countries? Globalization and Health, v. 4, n. 8, pp. 1-10. 


\section{SEGURANÇA OU INSEGURANÇA DA SAÚDE MUNDIAL NA ÁFRICA? MAIS SAÚDE PARCIAL DO QUE SAÚDE GLOBAL}

\section{DOMINIQUE KEROUEDAN}

Resumo: O presente ensaio aborda as três grandes condições de emergência da noção de saúde mundial, com especial referência à África: (i) o risco pandêmico e outros problemas sanitários comuns; (ii) as evoluções institucionais da ajuda ao desenvolvimento em saúde; e (iii) a genealogia de uma definição comum da saúde mundial. O texto discute os riscos potenciais para as populações mais pobres do planeta trazidos pela tentação de globalização que acompanha a noção de "saúde global", ainda e sempre às custas das pessoas mais frágeis, mais pobres, mais doentes e também menos favorecidas: as mulheres e as crianças. Os Estados e as instituições internacionais interestatais veem confiscada sua missão de serviço público, correndo o risco de fragilizar a soberania, a segurança e a paz nacional e internacional que as instituições públicas e privadas norte-americanas supostamente visavam proteger.

Palavras-chave: África; Cooperação Internacional; Desenvolvimento; Saúde Global; Segurança.

\section{SECURITY OR INSECURITY OF WORLD HEALTH IN AFRICA? A RATHER PARTIAL THAN GLOBAL HEALTH}

Abstract: The present essay addresses the three major conditions for the emergence of a world health notion, with special reference to Africa: (i) the pandemic risk and other common sanitary issues; (ii) the institutional evolution in health development aid; and (iii) the genealogy of a common definition for world health. This text discusses the potential risks for the poorest populations in the world brought about by the globalization temptation, which follows the notion of "global health," still and ever at the most fragile, the 
poorest, the sickest and least privileged people's expense: women and children. States and international interstate institutions have seen their public service mission being confiscated, running the risk of undermining sovereignty, security and national and international peace that North American private and public institutions allegedly aimed to protect.

Keywords: Africa; International Cooperation; Development; Global Health; Security.

Recebido em: 28/3/2015 Aprovado em: 17/6/2016 